\title{
An Analysis of Buzzwords in A Bite of China from the Perspective of Language Memetics*
}

\author{
Ling Li \\ School of Foreign Languages \\ Hubei Engineering University \\ Xiaogan, China
}

\begin{abstract}
The broadcasting of food documentary A Bite of China has triggered a sensation. Meanwhile, its language style is socially acknowledged and widely spread in the internet. The paper is centered on the internet buzzwords of A Bite of China. In order to reveal the propagation rules of internet buzzwords, the replication and propagation process of its internet buzzwords is discussed. Additionally, reasons for strong internet memes are also explored from the perspective of language memetics.
\end{abstract}

Keywords-Memes; language memetics; language style of A Bite of China; internet buzzword

\section{INTRODUCTION}

In 2012, the premiere of a food documentary, A Bite of China, has triggered an audience climax with the debut of CCTV's integrated channel. Afterwards, with the help of the new media, the movie's click-through rates have been so high that it has spawned a phenomenon of A Bite of China buzzwords in a very short time. Netizens are so crazy to imitate the subtitles of the documentary that there is a wide range of originative and funny creations like "A Bite of mother school", "A Bite of instant noodles", "A Bite of Chongqing", etc. Such imitations fully satisfy netizens' creating and teasing desires. Internet buzzwords sometimes are called memes, which are frequently used, widely spread and with overnight fame. They spread quickly just like viruses. Functionally, they express people's feelings and record social process. What's more, they reflect netizens' attitudes and assessments of public events or social phenomena. Memes are not produced directly from internet culture, therefore, the epidemic spread of memes are explicable.

\section{LANGUAGE MEMETICS}

\section{A. Memetics}

The word "meme" comes from Greek "mimeme", which means to imitate. In 1976, British ecologist Richard Dawkins first put forward the term "meme" in his book The Selfish Gene, then Chinese scholar $\mathrm{He}$ ziran introduced and translated it into "mo yin". According to Dawkins, memes

*Sponsored by Humanities and Social Science Youth Project-Study on the Construction Mode of Multi-modal Discourse in Chinese Food Culture, Board of Education, Hubei (15Q211) are similar to genes. Genes reproduce through heredity, while memes propagate through imitation. Memes are basic units of cultural transmission. Memes are presented in many forms like melodies, concepts, idioms, clothes, architecture, etc. The key element in a meme is to imitate. According to the range, time and productivity, memes can be divided into two categories, that is, strong memes and weak memes. Strong memes are featured with vitality, productivity and widespread transmission. As a matter of fact, strong memes survive in the fierce competition of reproduction and transmission. Instead, weak memes are often short-lived and they gradually fade away with the social development.

Appearance of the new concept "meme" has attracted wide attention from scholars both at home and abroad. Thus, the term "meme" has been introduced into many disciplines. In fact, languages and memes are closely related; language is just a carrier of a meme. Memes survive on the basis of imitating, reproducing and propagating hosts, thus limitless buzzwords are formed. Memes not only depend on languages, but also promote the developments of languages. In a word, the theory of memes is a new way to explain cultural evolution and it also provides a new research perspective for languages and cultural phenomena.

\section{B. Types of Language Memes}

Generally, the replication and propagation mode of language memes can be divided into two types, one is memegene transmission and the other is meme-behavioral transmission. Genetic memes directly transmit the information, in the same or different forms. Information to be transmitted often includes various kinds of quotations, slogans, famous sayings and so on. Some information are transmitted in the same form, for example, political leaders often copy Zhu geliang's (a famous political leader in ancient China, in the Three kingdoms period) saying "devotion to duty and death" to express loyalty to the country. Besides, expressions like "study hard and make progress everyday", "harmonious society" and "Chinese dream" are often used in appropriate occasions. On the other hand, meme-behavioral transmission refers to the expression of different contents but in the same forms. In the process of transmission, the forms of original information are kept but contents are replaced with new information. For example, the propagation of buzzwords in A Bite of China is a typical meme-behavioral transmission. At first, A Bite of China is 
only accepted by people as a kind of food information, which infects the audiences. Then people understand, accept and store it in mind. Finally, audiences are assimilated by the information. The memory process is reflected in the process of staying in the hosts' brain. While in communication, the meme A Bite of China is singled out from the storage and forming the material shape that can be perceived through discourse. This is the stage of expression. As for the universal internet, the language style of A Bite of China is quickly spread, its original connotation is constantly expanded and more creative expressions are being swept over the internet. Therefore, a research of strong language memes in A Bite of China is both quite helpful to cultivate correct attitudes to internet buzzwords and very necessary for a good grasp of rules of using memes in internet culture.

\section{REASONS FOR STYLE “A BITE OF CHINA” BEING STRONG MEMES}

The popularity of language styles in A Bite of China is not only a reflection of Chinese netizens' creativity of language symbols, but also a representation of netizens' social psychology, What's more, it gathers public opinions from netizens.

\section{A. Presenting the Beauty of Language Variation}

As society develops, language usage also changes a lot. In the internet era, networked communicative media has changed people's ways of thinking and communication. The virtual and open internet context breaks traditional pattern of communication, especially in the language use. The network language does not obey the inherent norms of language usage any longer, thus it leads in language variation. Internet language has accelerated language variations, thus language variations are quite popular in the internet. Language variations often include two kinds, namely, form variation and content variation. They are mainly featured with creation.

The unique subtitles in A Bite of China have been highly praised by netizens as ideal expressions just like perfect composition of the college entrance exam. Creative and originative expressions break traditional norms of language usage and also present the beauty of language variation. These expressions are the artistic presentation of languages and they fully reveal the beauty of language. For example, a paragraph of classical expressions are used to describe the common process of drying fish. "Brilliant sunshine communicates with fresh fishes thoroughly; this is an agreement between people and heaven." The expressions are originally aimed at describing the food-production process, but after using the language style of A Bite of China, all the descriptions become extraordinarily meaningful. What's more, the documentary also adopts bright words, poetic expressions and harmonious conception to describe daily food activities-eating melon seeds. "Melon seeds undoubtedly witness true love between sun and sunflower. Mutual understandings and cooperation between lip and fingers make people's enthusiasm burst out and shattered, leaving only the ordinary smell and empty shells." And there is also a classical description of making Zongzi: "Young people from all over China are protecting the vitality of traditional food by hand." Generally, if something or someone is protected by hand, it must be concrete, however, "protected by hand" is collocated with the abstract word "vitality". The unusual usage produces strong artistic effects and highlights tensions in languages and reminds people of the mission of protecting and inheriting Chinese food traditions. Attracted by literary and artistic subtitles in the documentary, netizens constantly imitate and reproduce the similar language style. For example, a Hubei netizen created a description about the local food, Hankou Style Noodle.

Hankou Style Noodle, a kind of typical breakfast for local people in Wuhan, witnesses true love between flour and sesame paste. After processes of half-boiling, cooling, stirring and mixing, noodles and sesame paste are appropriately melded. The seemingly ordinary bowl of noodles instantly radiates unparalleled fragrance and tastes. It prepares the diligent workers with positive energy.

It is quite obvious that the language creation of $A$ Bite of China often tests the creators' language skills and literary accomplishments. It is not just simple imitation, but delicate creation.

\section{B. Satisfying the Psychological Needs of Netizens}

Internet provides netizens with a wide and free platform for communication, internet language has become an important tool for netizens to express opinions, exchange and communicate with each other. The buzzwords in "A bite of China" satisfy public demands of participation and expression in network events. Compared with traditional language expressions, originative and interesting expressions are favored more by netizens. In the documentary, styles of describing daily trivialities by using artistic expressions just meet netizens' needs of following the trend and seeking for unconventional things. Daily chores like eating instant noodles, being bitten by mosquitoes, washing dishes, etc. become funny after being described in the style of A Bite of China. For example, an investor artistically describes the scene of eating instant noodles after stock market's closing: The stock market closed, Liu turned off the computer, prepared a bowl of instant noodles with boiling water. Chinese stock investors prefer to enjoy the unique delicacy in the dark, because they believe that only in this way can sadness of buying at the top and selling at the bottom can be get rid of.

Network language is not only a psychological symbol, but also the result of interaction between hot issues and netizens' psychology. Netizens intentionally imitate and reproduce in order to seek for a sense of belonging and recognition, which can be easily felt and strengthened. Internet buzzwords are considered as a "weapon of the weak". Therefore, users' intentions and attitudes to buzzwords vary a lot. Some use buzzwords for the pursuit of fashion and fun, some for getting rid of boredom and some for joking. For example: a netizen created a description of biting by mosquitoes by imitating the language style of $A$ Bite of China. Summer is a harvest season. A young girl, smooth and clean skin, fresh blood, all these perfectly satisfy mosquitoes' appetite in a pleasant summer day. For 
mosquito A Shan, it is also the golden moment in a year to enjoy the precious gifts of nature. Such an expression shows the creator's humor and ridicule of traditional elegant and classical ways of description.

From the above mentioned examples, internet buzzwords are in essence fragmented expressions. Meanwhile, those expressions also reflect netizens' psychology. The popularity of buzzwords in A Bite of China just reveals netizens' understandings of current events and reflects their practical emotions.

\section{Conforming to the Features of Network Transmission}

According to British scholar Kate Distin, successful memes must have five elements, namely, convenient channels of network language transmission, interactivity of network environment, convenience of storage environment, availability of communicative tools and rich content of transmission.

As an indispensable carrier of transmission, internet serves as a precondition for the spreading of buzzwords in $A$ Bite of China. The popularity of the documentary can not only be attributed to the influences of traditional media but also the powers of network transmission. In order to provide a more convenient platform for audiences, an official website of the documentary was established by China network TV station. Additionally, IQIYI became the first to sign a contract with CCTV to buy the copyrights. The premiere day of the documentary has aroused concerns of social networks such as Microblog and BBS. Afterwards, it became a hot topic for netizens.

Internet is the home of network languages and it leaves room for group transmission. As long as the internet is available, netizens can receive and send information anywhere. Once a piece of news occurs, it will be spread out crazily via the internet. The speed and scope of internet transmission is beyond imagination.

Internet buzzwords develop quickly and their contents have been gradually enriched. Obviously, there are various kinds of creations concerning buzzwords of A Bite of China and most of them appear in different ways like news comment, microblog, virtual community, QQ, Wechat, etc. In the era of internet, information is transmitted in all directions by imitating, reproducing and transmitting. On the one hand, language styles in the documentary are interpreted by netizens; on the other hand, the connotation and extension of internet buzzwords also changes accordingly in the dynamic context.

Memes speed up the transmission of network language. Internet buzzwords in A Bite of China follow the rules of meme transmission. According to its unique life circle and selection criteria, language memes constantly grow in the process of self-replication, mutual replication, innovative replication and creative combinations. Because of advantages in fidelity, fertility and longevity, buzzwords in $A$ Bite of China have become strong language memes and show vitality.
Buzzwords in A Bite of China become tools for internet communication. Unlike traditional ways of communication, there are no obvious differences between subject and object. Members of group transmission vary a lot and contents of transmission also differ in forms. Various kinds of internet languages are reflections of different group members. Meantime, internet languages also promote the formation of communicative groups and circles. By group transmitting, the scope of internet languages is broadened.

\section{CONCLUSION}

Endless strong language memes of A Bite of China is a product in the era of internet. Netizens use internet buzzwords to transmit information, communicate with each other, express feelings and seek for self-recognition. The theory of language memes can be used to explain many linguistic and cultural phenomena. As a matter of fact, it provides a favorable perspective for us to understand and grasp the popularity and transmission of buzzwords in A Bite of China.

\section{REFERENCES}

[1] Ma ruohong, The Unique Language Style of A Bite of China, Language Planning, 10(2014) 61-62.

[2] He Ziran, Memes in Languages, Linguistic Sciences, 6(2005)63.

[3] Distin, K., Cultural Evolution. Beijing: Word Book Publishing Corporation. 2015.

[4] Dawkins, R., The Selfish Gene, Beijing: Science Press. 2014.

[5] Cao Jin, Jin Yan, Theoretical Interpretation on the Communication Power of Internet Strong Language Memes, Journal of International Communication, 2 (2016) 37-56. 